\title{
Estudio sobre el uso de la gamificación en plataformas de e-learning: teorías de comportamiento, tasas de participación y experiencias de uso
}

\section{Study on the use of gamification in e-learning platforms: behavioral theories, participation rates and usage experiences}

Vinicius Covas Alves*

Universidad Anáhuac México

Av. Universidad Anáhuac núm. 46, Col. Lomas Anáhuac, C.P. 52786, Huixquilucan, Estado de México

Editor: Rogelio del Prado Flores

Fecha de recepción: 27 de abril de 2020

Fecha de aceptación: 9 de mayo de 2020 vinicius.covas@anahuac.mx https://orcid.org/0000-0001-9948-2940

\section{RESUMEN}

Se entiende como gamificación la aplicación de elementos de juegos en ambientes ajenos que no son necesariamente de juegos con el fin objetivo de promover la motivación y participación de los usuarios. En el contexto educativo, se estimula de esa forma el proceso de aprendizaje. El uso de la gamificación en estos entornos podría ser una solución a las bajas tasas de participación y compromiso de los usuarios en plataformas como los MOOC.

Este análisis tiene la ambición de identificar experiencias de otros investigadores con respecto a la presencia de esta táctica en estos ambientes digitales, a través de una investigación documental y comparativa de acuerdo con sus experiencias, contrastando sus resultados y hallazgos, como también asociando las perspectivas teóricas para la investigación de la gamificación que proponen De Liu, Radhika Santhanam y Jane Webster (2016).

El objetivo es discutir la aplicación de elementos de gamificación en entorno al aprendizaje en línea; conceptualizar la dinámica de gamificación, destacar los hallazgos de

\footnotetext{
* Alumno del Doctorado en Investigación de la Comunicación por la Universidad Anáhuac México. Maestro en Dirección de Empresas de Entretenimiento por la misma universidad. Licenciado en Comunicación Social por la Universidad Veiga de Almeida, Brasil. Se interesa por estudios relacionados con comunicación, sociedad y cultura digital. Experto en desarrollo de marcas en un entorno digital. Conferencista internacional con participación en TEDx. Semifinalista del Premio Young Inspiring 20 I2 de la revista VEfA y de la Fundação Estudar.
} 
investigadores en cuanto a esta temática, las perspectivas teóricas de la gamificación y su relación con la motivación y compromiso de usuarios.

Como expectativas de resultados, se espera que podamos generar un mejor entendimiento de la literatura sobre el uso de la gamificación, siendo este un mecanismo de disparo de motivación y compromiso de los usuarios en plataformas de e-learning.

Palabras clave: gamificación, e-learning, teorías de comportamiento, usuarios.

\section{ABSTRACT}

Gamification is understood as the application of elements of games in external environments that are not necessarily games in order to promote the motivation and participation of users. In the educational context, the learning process is stimulated in this way. The use of gamification in these environments could be a solution to the low participation rates and commitment of users on platforms such as MOOCs.

This analysis has the ambition to identify experiences of other researchers regarding the presence of this tactic in these digital environments, through documentary and comparative research according to their experiences, contrasting their results and findings, as well as associating theoretical perspectives for the gamification research proposed by De Liu, Radhika Santhanam and Jane Webster (20I6).

The objective is to discuss the application of gamification elements in an online learning environment; conceptualize the dynamics of gamification, highlight the findings of researchers regarding this topic, the theoretical perspectives of gamification and its relationship with the motivation and commitment of users.

As expectations of results, it is expected that we can generate a better understanding of the literature on the use of gamification, this being a trigger mechanism for motivation and commitment of users in e-learning platforms.

Keywords: gamification, e-learning, behavior theories, users.

\section{INTRODUCCIÓN}

$\mathrm{E}^{n}$ n un mundo de la información tecnológicamente globalizado las competencias son exponenciales. Durante la fase del Internet considerada Web 2.0, aparecen variados sistemas de tecnologías que facilitan la emisión, el acceso y el tratamiento de la información, como las redes sociales, wikis, blogs y ambientes de aprendizaje digital como los llamados Massive Open Online Course, conocidos por la sigla MOOC, que popularmente se considera 
una herramienta fundamental en el proceso de democratizar el acceso a la educación global y que contribuye con el desarrollo de profesionales que están en países en vías de desarrollo.

Se considera que los Massive Open Online Courses poseen una gran capacidad y valor en cuanto a la ampliación y acceso a una fuente de conocimiento y, por lo tanto, son vistos como una de las principales herramientas en la democratización de la educación en un contexto global.

Con los pronósticos de la coyuntura global en cuanto a la demanda por el acceso a la educación, que para los próximos años aumentará de manera considerable en economías emergentes, en algunas zonas existen restricciones en cuanto al acceso a dispositivos de tecnología, infraestructuray apropiación de parte de los usuarios a plataformas como los MOOC.

Para agregar aún más valor a la importancia de las plataformas como los MOOC en un escenario futuro en cuanto al desarrollo de una educación sustentable global, de acuerdo con el UIS Fact Sheet de la UNESCO publicado en octubre de 20I6, para proporcionar educación a todos los niños al año de 2020, los países necesitan reclutar alrededor de 9.8 millones de maestros de primaria y 22.3 millones de maestros de secundaria. Y para el año de 2025, estas cifras aumentan en I7.8 millones para educación primaria y 33.5 millones para la educación secundaria. De esta forma, el mundo tiene una demanda de casi 69 millones de nuevos maestros para alcanzar las metas educacionales para el año de 2030.

Por otro lado, los Massive Open Online Course (MOOC), como su propio nombre ya dice "Massive", tienen la capacidad de distribuir y alcanzar una audiencia masiva de estudiantes que podrían tener acceso a una educación global a un clic. En el año de 20I8, de acuerdo con Class Central (20I8), tuvimos la suma de Ior millones de estudiantes inscritos en cursos en línea y más de 900 universidades con cerca de II 400 cursos disponibles. Las principales plataformas son: Coursera con entorno de 37 millones de estudiantes; edX con I 8 millones; XuetangX con I4 millones; Udacity con Io millones y FutureLearn con 8.7 millones de alumnos registrados.

La gran mayoría de los cursos a disposición pertenecen a la categoría de tecnología y business, seguido por ciencias sociales, humanidades y ciencia. Estas poseen tamaña relevancia en el escenario de desarrollo social y económico, y si lográramos que más estudiantes se especialicen en estos campos podríamos de alguna forma empezar a percibir el beneficio de una formación en esta modalidad.

La tecnología es determinante para el desarrollo de una nación. Es lo que destaca el Foro Económico Mundial (20I8) que apunta que “la adopción de tecnología es un indicador clave del desarrollo económico" y destaca que los países necesitan desarrollar sistemas de tecnologías de información y comunicación que puedan permitir nuevos modelos de colaboración y aumentar la eficiencia y productividad de sus economías. 


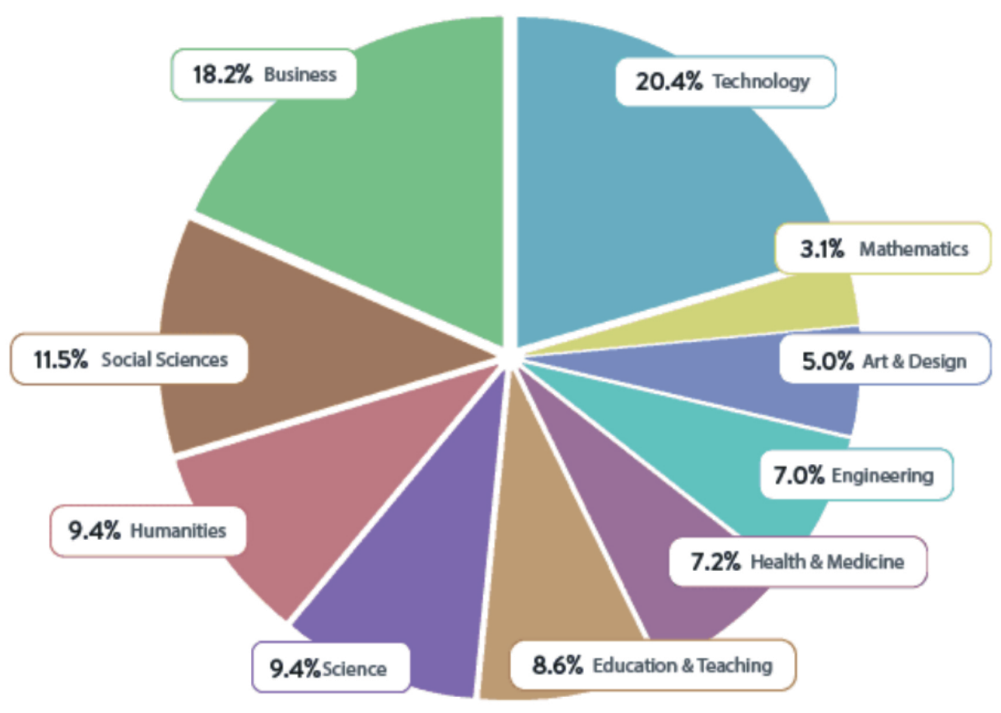

FUENTE: DHAWAL SHAH (2018). https://www.classcentral.com/report/mooc-stats-2018/

En estos días, acceder a servicios básicos de salud, educación y financieros es parte de un proceso informacional donde la tecnología tiene gran responsabilidad en ello, y así se cree que dichas plataformas de conocimiento en línea son claramente una alternativa para el desarrollo de estudiantes, profesionales y emprendedores que a partir de ahora poseen una competencia global.

Sin embargo, aunque tengamos números que despiertan la atención en cuanto a propagación de este adviento en aprendizaje y desarrollo de profesionales, la realidad es un poco más compleja.

Los MOOC están fallando en cuanto a su misión de lograr una "democratización" del conocimiento e incluso podría aumentar el gap en el acceso al conocimiento, contribuyendo de alguna forma con el aumento de la brecha digital impactando aún más las minorías. Esto debido a que los estudiantes que logran concluir un curso en la plataforma son en su mayoría usuarios que tienen una licenciatura o posgraduación, y gran parte son hombres en regiones consideradas desarrolladas del mundo. Pero para no entrar en una cuestión social demográfica y enfocarnos el tema de este artículo, hablaremos un problema de igual tamaño e importancia que está sucediendo en esas plataformas. 
Coursera es la plataforma de mayor relevancia en el actual escenario con cerca de casi 40 millones de estudiantes registrados. Aunque el número sea considerado alto, una pequeña parcela de estos usuarios logran mantenerse activos en el correr del curso.

Hill (20I3) identificó los tipos de usuarios que participan en Coursera y encontró cinco categorías de usuarios, los que no se presentan, los observadores, los que desertan, los participantes pasivos y los participantes activos, encontrando diferentes niveles de colaboración de estos usuarios. Engle (2014) encontró que casi la mitad de los inscritos a un curso masivo como Coursera no participan de manera activa, y entre 5 y $10 \%$ de los que participan al menos en una actividad en la plataforma lo completan de manera éxitosa, logrando obtener un certificado. En 2015, HBR encontraría que $4 \%$ de estos efectivamente concluyen un curso.

Necesitamos plantear preguntas de investigación sobre cómo podemos contribuir con este problema de la deserción de los estudiantes en tales plataformas, identificando elementos de multidisciplinares que podrían favorecer las tasas de retención y de alguna forma hacer que los usuarios que se inscriban en las plataformas se mantengan activos hasta la conclusión de sus cursos.

\section{OBJETIVO}

Los MOOC se presentan como un atractivo solucionador para un problema de escala global y que cada vez más parece ser conveniente que se busquen soluciones. La democratización del acceso al conocimiento y una enseñanza en un contexto social masivo son algunas las múltiples ventajas de los MOOC (Dillenbourg, Fox, Kirchner, Mitchell y Wirsing, 20I4).

Con motivos de este artículo, se tiene por objetivo investigar cómo los procesos conocidos como gamificación están siendo usados en este escenario de mejoría de uso de los MOOC para la educación, contextualizando los diferentes tipos de aplicaciones ya experimentados como un beneficio a los educadores, diseñadores instruccionales y programadores.

\section{METOdología}

Este artículo fue trabajado a través de una investigación documental de estudios que relacionan el uso de elementos de gamificación en plataformas de $e$-learning. El análisis fue realizado de manera individual y la intención era identificar las experiencias realizadas en el campo y correlacionar las teorías sociales por detrás de la gamificación. 
Se procedió a una inspección sobre estos trabajos y, tras obtener los resultados de dichas investigaciones, se desarrolló una conclusión con comentarios interpretativos sobre el impacto de la gamificación en estos ambientes.

Para un análisis comparativo, se construye una tabla que contrasta las investigaciones de los autores.

\section{RESULTADOS}

En los últimos años, investigadores han llamado cada vez más la atención sobre la implementación de estrategias de gamificación para involucrar a los estudiantes y promover el propósito de aprendizaje de los estudiantes (e.g. Barata, Gama, Jorge, y Gonçalves, 20I3; Domínguez, Saenz-de-Navarrete, De-Marcos, Fernández-Sanz, Pagés, y Martínez-Herráiz, 20I3; Ibanez, Di-Serio, y Delgado-Kloos, 20I4).

No es solo característico de los MOOC sino también de otras plataformas en diferentes industrias, uno de los principales problemas es lo que está asociado con la motivación y las variables que hacen que un usuario se registre o no a algo y una vez registrado que se mantenga usando el servicio ofrecido. Como se ha comentado, aunque sean muchos los registrados a los cursos MOOC las tasas de abandono son muy elevadas (Alario-Hoyos, Pérez-Sanagustín, Delgado-Kloos, Parada Gélvez, y Muñoz-Organero, 20I4; Gütl, Rizzardini, Chang y Morales, 20I4; Jordan, 20I4; Poy y Gonzales-Aguilar, 2015). Es de conocimiento que el abandono y la falta de motivación en el proceso de aprendizaje es un problema significativo que muchas escuelas y educadores enfrentan. Numerosos estudiantes carecen de motivación e interés para aprender (Zichermann y Cunningham, 20II).

Investigaciones entorno de este asunto han sido desarrolladas desde hace décadas pasadas.

Bonwell y Eison (I99I) desarrollaron una serie de estrategias con la intención de promover un proceso de aprendizaje más relevante y entre sus resultados está el uso de juegos en el método de enseñanza, lo que podemos ver como la adopción de mecanismo de gamificación. Aunque sea una propuesta que se adopta en gran parte en el ambiente de educación presencial desde hace mucho, como por ejemplo la asignación de puntos, calificaciones, grados y los más variados modelos de recompensa, los conceptos de gamificación también empiezan a aparecer en contextos masivos de enseñanza con el objetivo de mejorar los resultados de aprendizaje, las tasas de retención e interacción entre los miembros.

\section{LA GAMIFICACIÓN}


Podemos entender como gamificación el uso de elementos y sistemas que son provenientes y típicos de los juegos en contexto considerados no recreativos (De Sousa Borges, Durelli, Reis y Isotani, 20I3; Deterding, Dixon, Khaled y Nacke, 20II). Una mecánica basada en el juego que hace el uso del diseño y pensamiento que se encuentra en juegos para involucrar a las personas y motivarlas a la acción, promoviendo aprendizaje y resolviendo problemas (Kapp, 2012).

El contexto y el diseño de estos procesos de gamificación en los juegos son determinantes para eléxito de su aplicación (Dichev y Dicheva, 20 17; Dicheva, Dichev, Agrey Angelova, 2015; Hamari, Koivisto y Sarsa, 2014).

\section{AMBIENTE COLABORATIVO}

El incremento de la motivación de los usuarios en estos ambientes puede estar relacionada con los impulsos a la colaboración y vinculación de los estudiantes con las actividades del MOOC.

Aunque haya estudios que confirman todos los potenciales beneficios posibles con la aplicación de estos elementos en ambientes de aprendizaje en línea, el uso de la colaboración (Manathunga y Hernández-Leo, 2015; Margaryan, Bianco y Littlejohn, 2015) y la gamificación (Dicheva et al., 2015; Ortega-Arranz, Muñoz-Cristóbal, Martínez Monés, Bote-Lorenzo y Asensio-Pérez, 20I7a) es considerada todavía limitada, con pocas experiencias de uso, quizá por una incapacidad tecnológica de las herramientas disponibles o por falta de planeación estratégica en el desarrollo y planteamiento de los cursos puestos a disposición. La heterogeneidad de los perfiles de los alumnos también podría ser una de las variables que afectan esta dificultad de proponer diseños pedagógicos participativos y con un grado hacia la gamificación que se encuadra con los diferentes tipos de usuarios y los factores únicos que a cada uno se suman.

En el artículo "Uso de la colaboración y la gamificación en MOOC: un análisis exploratorio” de Sara García-Sastre, Miriam Idrissi-Cao, Alejandro Ortega-Arranzy Eduardo Gómez-Sánchez de la Universidad de Valladolid, España, los autores realizaron una revisión y análisis de la literatura disponible para identificar si se fomenta el uso de la gamificación en los MOOC y observaron alrededor de 20 de ellos, para así entonces representar cómo estos cursos lidian con la implementación de elementos de gamificación y colaboración en sus diseños didácticos.

Los autores diseñaron una tabla con los 20 cursos analizados y asignaron si cumplen o 
no con algunas de las herramientas que ellos consideraban que pudieran contribuir con un sentido colaborativo o de gamificación.

TABLA 1. USO DE LA COLABORACIÓN Y LA GAMIFICACIÓN EN MOOC. UN ANÁLISIS EXPLORATORIO

\begin{tabular}{|c|c|c|c|c|c|c|c|c|c|c|c|c|c|c|c|c|c|c|c|c|c|}
\hline \multicolumn{2}{|c|}{$\mathrm{MOOC}$} & 01 & 02 & 03 & 04 & 05 & 06 & 07 & 08 & 09 & 10 & 11 & 12 & 13 & 14 & 15 & 16 & 17 & 18 & 19 & 20 \\
\hline \multirow{6}{*}{ Motivación } & Obligatorio & & & $x$ & & & & $x$ & & & & & & & & & & & & & \\
\hline & Espontáneo & & $x$ & & & & & $x$ & $x$ & $x$ & $x$ & & & $x$ & $x$ & & & & $x$ & $x$ & $x$ \\
\hline & $\begin{array}{l}\text { Enriquece el } \\
\text { contenido }\end{array}$ & $x$ & $x$ & & $x$ & $x$ & & $x$ & & $x$ & $X$ & & & $X$ & $x$ & & & & $x$ & $x$ & \\
\hline & Resolver tareas & & & $x$ & $x$ & $x$ & & $x$ & & $x$ & $x$ & $x$ & & & & & & $x$ & & & \\
\hline & $\begin{array}{l}\text { Beneficio } \\
\text { indirecto }\end{array}$ & & & $x$ & & & & & & & & & & & & & & & & & \\
\hline & Desconocido & & & & & & & & & & & & & & & & & & & & \\
\hline \multirow{3}{*}{ Escala } & Gran grupo & $x$ & $x$ & $x$ & $x$ & & & $x$ & $x$ & $x$ & $x$ & $x$ & $x$ & $x$ & & & & $x$ & & & \\
\hline & Grupo medio & $x$ & & & & & & & & $x$ & & & & & & & & & & & \\
\hline & Pequeño grupo & $x$ & & & & & & & & $x$ & & & & & & & & & & & \\
\hline \multirow{4}{*}{$\begin{array}{l}\text { Relevancia de la } \\
\text { colaboración para } \\
\text { el aprendizaje }\end{array}$} & Fundamental & & & & & & & & & & & & & & & & & & & & \\
\hline & Ocasional & & $x$ & $x$ & $x$ & $x$ & & $x$ & $x$ & $x$ & $x$ & $x$ & & & & & & $x$ & & & \\
\hline & Anecdótico & $x$ & & & & & & & & & & & & $x$ & $x$ & $x$ & $x$ & & $x$ & $x$ & $x$ \\
\hline & Inexistente & & & & & & $x$ & & & & & & & & & & & & & & \\
\hline \multirow{7}{*}{ Recursos } & Foros & $x$ & $x$ & & $x$ & & & $x$ & $x$ & $x$ & $x$ & & & & & & & & & & \\
\hline & Chats & & & & $x$ & & $x$ & $x$ & $x$ & $x$ & $x$ & & & & & & & & & & \\
\hline & Redes sociales & & & & & & & & & & $x$ & & & & & & & & & & \\
\hline & Wikis & & & & & & & & $x$ & & & & & & & & & & & & \\
\hline & Blogs & & & & & $x$ & & & & & & & & & $x$ & & & & & & \\
\hline & Hangouts & & & & & & & & $x$ & $x$ & $x$ & & & & & & & & & & \\
\hline & Otras & $x$ & & $x$ & $x$ & $x$ & & $x$ & $x$ & $x$ & & & & & $x$ & $x$ & & $x$ & & & \\
\hline \multirow{2}{*}{$\begin{array}{l}\text { Evaluación } \\
\text { corporativa }\end{array}$} & Por pares libre & & & & & & & & & $x$ & $x$ & $x$ & & & & $x$ & & $x$ & & & \\
\hline & $\begin{array}{l}\text { Por pares con } \\
\text { rúbrica }\end{array}$ & $x$ & $x$ & $x$ & $x$ & $x$ & & $x$ & & $x$ & $x$ & & & $X$ & $x$ & $x$ & $x$ & $X$ & & & \\
\hline
\end{tabular}

FUENTE: GARCÍA-SASTRE, S.; IDRISSI-CAO, M.; ORTEGA-ARRANZ, A.; GÓMEZ-SÁNCHEZ, E. (2018). 
De acuerdo con los autores, en ninguno de los 20 MOOC analizados se desarrollaban tareas en las que un aprendizaje colaborativo fuera el eje central de las actividades propuestas en los cursos, en el proceso de aprendizaje, aunque existan herramientas sociales, como los foros, wikis, chats y redes sociales. Los autores mencionan que las herramientas más utilizadas en los cursos analizados fueron las fotos y las redes sociales.

\section{MEDALLAS Y MECANISMO DE RANKING}

En cuanto a los elementos de gamificación en los 20 cursos analizados, se observó que en uno de ellos se otorgaron medallas como sentido de recompensa a los estudiantes que cumplían con ciertas actividades, lo que puede ser interpretado como una partícula motivadora con la intención de que completen los módulos e interactúen en los foros con otros estudiantes, profesores y demás miembros (Ortega-Arranz, Muñoz-Cristóbal, Martínez-Monés, Bote-Lorenzo y Asensio-Pérez, 20I7b).

Los considerados puntos, insignias y rankings de clasificación (Dicheva, Dichev, Agre y Angelova, 2015; Hamari et al., 2014) son los elementos más comunes en ambientes de gamificación. Podemos entender cómo las insignias, medallas, trofeos, íconos, logotipos e imágenes podrían traducir la idea de obtención de un logro, de cumplimiento de una actividad con éxito (Bunchball, 2o Io; Educause, 20II). Los rankings, por así decir, son presentados en formato de tabla de clasificación y hacen una referencia a lista de puntaje que indican desempeño de un individuo en relación a otro (Bunchball, 20I0; Educause, 20II).

En otro curso, las medallas eran dadas para los estudiantes que completaron actividades de revisión entre pares o por participar en los foros comentando en las respuestas de otros estudiantes. La incorporación de un cuadro para rankear a los usuarios fue encontrada y ahí se compara el nivel de desarrollo de un estudiante con los demás, lo que despierta un sentido de competencia que podría conllevar hacia un efecto motivador.

En los análisis realizados por estos autores, se entiende que la gamificación apenas se utiliza como motivo de impulsar una participación más allá de las que se ve en las fotos y para motivar la revisión entre pares. 
TABLA 2. USO DE LA COLABORACIÓN Y LA GAMIFICACIÓN EN MOOC. UN ANÁLISIS EXPLORATORIO

\begin{tabular}{|c|c|c|c|c|c|c|c|}
\hline \multicolumn{2}{|c|}{$\mathrm{MOOC}$} & 01 & 05 & \multicolumn{2}{|c|}{0714} & \multicolumn{2}{|c|}{1620} \\
\hline \multirow{5}{*}{ Finalidad } & Motivación & $x$ & & $x$ & & & $x$ \\
\hline & Fomentar comportamientos & & & $x$ & $x$ & & \\
\hline & Mejorar aprendizaje & $x$ & & $x$ & & & \\
\hline & Otros & & & & & $x$ & \\
\hline & Desconocido & & $x$ & & & & \\
\hline \multirow{15}{*}{ Mecánicas y dinámicas } & Puntos & & & & & & \\
\hline & Niveles & & & & & & \\
\hline & Leaderboard / Rankings & & & $x$ & & & \\
\hline & Gifts & & & & & & \\
\hline & Medallas & $x$ & $x$ & $x$ & $x$ & $x$ & \\
\hline & Temporizadores & & & & & & \\
\hline & Vidas & & & & & & \\
\hline & Objetivos virtuales canjeables & & & & & & \\
\hline & Narrativa & & & & & & \\
\hline & Misiones & & & & & & \\
\hline & Competición & & & & & & \\
\hline & Colaboración & & & & & & \\
\hline & Desbloqueo de contenidos & & & & & & \\
\hline & Elementos sociales & & & & & & \\
\hline & Otros & & & & & & $x$ \\
\hline \multirow{2}{*}{ Tipo } & Individual & $x$ & $x$ & $x$ & $x$ & $x$ & $x$ \\
\hline & Colectivo & & & & & & \\
\hline \multirow{3}{*}{ Herramientas } & Las de la plataforma MOOC & & & & & & \\
\hline & Externas & & & & & & \\
\hline & Ambas & & & & & & \\
\hline
\end{tabular}

FUENTE: GARCíA-SASTRE. S.; IDRISSI-CAO, M.; ORTEGA-ARRANZ, A.; GÓMEZ-SÁNCHEZ, E. (2018). 


\section{TECNOLOGÍA}

Sobre la tecnología que se ocupa en cada tipo de curso, Sara García-Sastre, Miriam Idrissi-Cao, Alejandro Ortega-Arranz y Eduardo Gómez-Sánchez observan que con alguna frecuencia se utilizan herramientas externas a los MOOC para gestionar los elementos de juegos, aunque las actividades gamificadas ocurran dentro de las plataformas. Se identifica el uso de Open Badges como una de las herramientas que permiten incrustar información de un curso y exportarla para el manejo de la dinámica de gamificación. Otra herramienta identificada es Karma, en OpenMooc. Badgr también es otra que permite integrar a los MOOC para realizar actividades de gamificación.

Tras analizar los 20 MOOC seleccionados, los autores destacan que los formatos de estos cursos han ido avanzando, aunque de forma tímida, hacia una incorporación de "pedagogías activas" y que se encuentran actividades que potencializan la interacción social entre los usuarios, lo que es un atractivo para la construcción de una percepción de comunidad entorno del tema estudiado y que podría contribuir con la retención de los estudiantes. Según ellos, los MOOC que adoptan elementos de gamificación pueden demostrar alguna evidencia de ser un elemento motivador que afecta al alumno y que pueden evitar el abandono del curso.

Para Domínguez (2013), la gamificación podría llegar a obstaculizar la participación de los estudiantes. Barata (2013) cree que la gamificación puede tener efectos positivos en los estudiantes. Adentrando a las herramientas, como por ejemplo el uso de las insignias en estos ambientes educacionales, un estudio concluyó que no había evidencia suficiente para poder afirmar que el uso de la insignia podría mejorar el compromiso de los estudiantes en un sistema de aprendizaje y que este podría impactar la efectividad de aprendizaje (Falkner y Falkner, 20I4). Otro estudio buscó identificar el rol de elementos de la gamificación en nueve industrias (Hamari y col, 20I4). Como resultado, Hamari y col, argumentan que los estudios actuales se dispersaron, salieron de enfoque y hace falta una evidencia coherente sobre la efectividad de la gamificación en estos entornos. Necesitamos contestar la pregunta: ¿Los puntos, insignias y rankings podrían llegar a aumentar el compromiso cognitivo de los estudiantes?

Regresando a la referencia anterior al estudio de Barata y col (2013). El autor ha llevado a cabo un estudio de cinco años con la intención de identificar si al introducir elementos considerados provenientes de la gamificación era posible mejorar el performance en cuanto a la asistencia, participación y aprendizaje de estudiantes en un curso en línea. Los autores compararon los registros de participación en el foro, la asistencia al curso y las calificaciones de los primeros tres años de curso, que no tenían mecanismo de gamificación, y los dos años 
posteriores que ya tenían la inserción de mecanismos de gamificación. Como resultados, los investigadores declaran no haber encontrado un considerable aumento en la asistencia al curso por parte de los registrados, sin embargo, sí un crecimiento considerable en las publicaciones en el foro. Los investigadores también encontraron que el puntaje final promedio de los estudiantes que realizaron sus estudios en ambientes gamificados han tenido calificaciones mayores que los estudiantes que hicieron el curso cuando todavía no tenía elementos de gamificación. Se indica que otras variables pueden haber impactado los resultados, como material de apoyo, exámenes e instructores diferentes en las temporalidades en las cuales se realizaron los análisis.

Los autores Seaborn y Fels (2015) revisaron alrededor de 32 estudios que se dedicaban a investigar cómo la utilización de elementos de gamificación digital impactaron de manera pedagógica los cursos en línea. De los 32 estudios, 20 presentaron resultados positivos que relacionan la gamificación con mayores niveles de motivación y compromiso en las plataformas. Los demás estudios indican resultados que no comprueban una mejoría en la participación de los estudiantes con la introducción de elementos del juego.

Para contextualizar este artículo observacional de gamificación aplicada a las plataformas de educación en línea, es clave hablar de las teorías de motivación. Por cuenta de que la gamificación todavía es vista como un sistema emergente en muchas industrias, hay pocos casos de éxito en cuanto a desarrollo de sistemas exitosos, lo que se deduce es que en el caso de la educación, el diseño y la implementación de los procesos de gamificación deben ser significativos para los usuarios para así lograr garantizar su efectividad (Domínguez et al., 20I3). Para Nicholson (20I2), para que un diseño de gamificación sea considerado efectivo, es importante que los diseñadores instruccionales y demás profesionales que están involucrados en este proceso, integren elementos que estén centrados en el usuario en el contexto que necesitan estar ajenos al juego.

En "Toward Meaningful engagement: a framework for design and research of gamified information systems”, De Liu, Radhika Santhanam y Jane Webster, desarrollaron una tabla con una muestra de perspectivas teóricas para la investigación de la gamificación.

\section{TEORÍAS COMPORTAMENTALES}

Desde una perspectiva de la disciplina económica comportamental, junto a la teoría de posibilidades, se entiende que personas toman decisiones basándose en las pérdidas y ganancias potenciales, asociándolo a un sistema de plataforma de conocimiento que podría ser la relación entre el tiempo invertido en una actividad y la capacidad de obtención de aprendizaje. 
A través de una perspectiva de sistemas informacionales con la teoría de las características de los medios, podemos entender la decisión que toman los usuarios en cuanto al consumo de información a través de determinado medio y sus señales (George et al., 20I3) y cómo este puede influir en el desempeño de la tarea a ser ejecutada (Daft et al., 1987).

La teoría cognitiva social considera que los humanos funcionamos como la consecuencia de una interacción dinámica de influencias personales, comportamentales y ambientales, y está muy relacionada con el sentido de la autoeficacia (Compeau y Higgins, 1995) y postula que al menos que las personas puedan creer que sus acciones pueden producir los resultados que desean, estas tienen pocos incentivos para actuar o perseverar ante las dificultades que se presentan.

Acotando esto en el caso de la tasa de retención de un curso en línea, esto podría estar relacionado con el sentido de la creencia o la falta de ella de algunos usuarios hacia sus capacidades para gestionar una plataforma digital o la falta de elementos de motivación para que un usuario avance en sus actividades obligatorias en un curso. Desde el ámbito del marketing, la teoría de los estímulos postula que cuando el entorno es deficiente en proporcionar la estimulación deseada necesaria, el usuario buscará una novedad, un cambio, y de la misma forma, cuando el ambiente proporciona más estimulación de la deseada, el individuo se comportará para reducir esta estimulación, de tal forma que el individuo siempre actuará para mantener un equilibrio entre los niveles de estimulación logrando un sentido óptimo para él, y cada quien tiene su nivel particular (Wablers y col, I990).

Desde el campo de la psicología, la teoría de flujo representa el grado de placer y participación en una actividad (Csikszentmihalyi, 1975) que abarca las percepciones del control del usuario, el enfoque de atención, despertar la curiosidad y el interés intrínseco (Webster y Martocchio, 1993). Y en esta teoría podemos ver la presencia de elementos de la gamificación. Se mejorará la experiencia de flujo del usuario cuando a este se presenta un desafío con una estimulación óptima. En el caso que sea una actividad exigente, esta podría producir un sentimiento de ansiedad, si es poco desafiante, genera una sensación de aburrimiento (Csikszentmihalyi, 1975).

Para Deci y Ryan (2000), la base por detrás de la teoría de la gamificación es la teoría de la autodeterminación (Deci y Ryan, 1985), que está constituida como un explicativo de la motivación humana y que se puede aplicar a variados ámbitos.

La teoría de la autodeterminación establece en qué medida las personas deciden involucrarse o no libremente en la realización de sus actividades, a partir de una serie de mecanismos psicológicos reguladores de la conducta y que trata de enfocarse más hacia la motivación autodeterminada, que está relacionada con la motivación intrínseca. Conductas 
no autodeterminadas están relacionadas con una motivación extrínseca, caracterizada por una falta de motivación.

Desde la perspectiva de la psicología social, con la teoría de comparación social, vemos que las personas tienen en su naturaleza una condición de siempre evaluar sus habilidades y lo hacen de igual forma a través de una comparación con los demás, y la tendencia es siempre compararse con personas que tienen mayores habilidades.

Con la teoría de autodeterminación en mente, Biyun Huag y Khe Foon Hew (2015) en "Do points, badges and leaderboard increase learning and activity: a quasi-experiment on the effects of gamification", postulan que un diseño de aprendizaje que hace el uso de la gamificación debe de poseer tres propiedades básicas, siendo estos: I) hacer que las actividades de aprendizaje sean significativas para los usuarios concediendo a él la autonomía para elegir sus metas y actividades de aprendizaje (Deci y Ryan, 2000; Nicholson, 20I2); 2) lograr promover la percepción de los estudiantes que el nivel de dificultad de la tarea está al mismo nivel de sus habilidades y hacer que perciban que sus competencias y sus capacidades crecen mediante sus participaciones en dichas actividades (Csikszentmihalyi, I978); 3) otorgarles una tabla de rankeo donde pueden comparar su desempeño con sus compañeros y hacer que los alumnos sientan que están conectados entre sí (Deci y Ryan, 2000; Festinger, 1954). En este sentido, la autonomía, propósito y dominio son elementos considerados esenciales para que una actividad sea más significativa para los participantes (Deci y Ryan, 2000).

Todavía en la disciplina de la psicología social, la teoría de normas e influencias sociales nos irá a mostrar que las personas cuando se identifican y se categorizan en determinado grupo social, su comportamiento será guiado por las normas de aquel grupo (Terry y Hoog, I996).

La sensación de diversión podría permitir un mejor aprendizaje (Prensky, 200I). Según Prensky, el objetivo de la diversión es crear la relajación y motivación, elementos que permiten al alumno asimilar el conocimiento más fácilmente, ya que así la motivación podría aparecer de manera más simple. El beneficio de la diversión es una de las principales razones por la que se ocupan elementos de gamificación en estos entornos (Swanson y Ferguson, 20I4), debido a este sentido de exploración, sensación de logro y recompensa al concluir un nivel por ejemplo (Zichermann, 20I0) y al incorporar estas características en un ambiente de aprendizaje podríamos lograr un aumento en el interés intrínseco (Lieberman, 2006). El juego puede no representar una presión sobre los alumnos para cumplir un requisito, menos resistencia en el alumno hacia una actividad (Hughes y Lacy, 20I6), y en estos entornos se presentan un sentido de "dificultad deseable" importante en el proceso de obtención de conocimiento, un esfuerzo mental que conduce a una comprensión más profunda. 
En "What Video Games Have to Teach Us About Learning and Literacy", Gee (2003) destaca que los elementos de juego deberían permitir que los estudiantes trabajen de forma más colaborativa y haya más trabajo en equipo. Para Zhang y Clear (2015) cualquier diseño instruccional que ocupa características de la gamificación necesita proporcionar una mayor colaboración entre los estudiantes, y destacan que aquellos que logran éxito ayudan con el surgimiento de conductas positivas de aprendizaje. Kim y col. (2015) declaró que cuando estudiantes pasan a ser jugadores, estos colaboran y participan en una actividad compartida y orientada al logro de objetivos.

Un estudio (Herzig et al., 2015) apuntó que los estudiantes que recibieron comentarios constructivos después de haber fallado en una actividad, pudieron experimentar una reacción positiva en lugar de sentirse fracasados. Para Gee (20I4), el fracaso en entornos gamificados puede ser entendido como un camino hacia el éxito.

En un entorno gamificado fallar no es retroceder, se ve como una oportunidad de aprender de los errores y así corregirlos (Hanus y Fox, 20I5), de manera que un curso que adopta estas características permite al estudiante jugador intentar realizar una actividad más de una vez hasta lograr el éxito.

A continuación, se presenta una serie de estudios consultados para la producción de este artículo y que enriquecen la comprensión sobre el paradigma del uso de la gamificación en plataformas de e-learning.

\section{TABLA CONSOLIDADA DE ESTUDIOS ENTORNO DEL USO DE \\ LA GAMIFICACIÓN EN PLATAFORMAS E-LEARNING}

\begin{tabular}{|c|c|c|}
\hline Bonwell y Eison & 1991 & $\begin{array}{l}\text { Procesos de aprendizajes que usan los mecanismos de } \\
\text { juego como instrumento de didáctica son más relevantes. }\end{array}$ \\
\hline $\begin{array}{l}\text { Dichev y Dicheva. Agre y Angelova. } \\
\text { Hamari, Koivisto y Sarsa }\end{array}$ & $\begin{array}{l}2017 \\
2015 \\
2014\end{array}$ & $\begin{array}{l}\text { Puntos, insignias, rankings de clasificación son elementos } \\
\text { más comunes en estos ambientes de gamificación. El } \\
\text { contexto y diseño son relevantes para el éxito. }\end{array}$ \\
\hline Kapp & 2012 & $\begin{array}{l}\text { El uso de las mecánicas de aprendizaje basadas en } \\
\text { juegos involucran y motivan a las personas a la acción } \\
\text { promoviendo el aprendizaje y resolución de problemas. }\end{array}$ \\
\hline $\begin{array}{l}\text { Sara García-Sastre, Miriam Idrissi- } \\
\text { Cao,Alejandro Ortega-Arranz, } \\
\text { Eduardo Gómez-Sánchez }\end{array}$ & 2018 & $\begin{array}{l}\text { Medallas y cuadros de ranking pueden elevar la motivación } \\
\text { y participación de los usuarios, ya que se estimula un } \\
\text { efecto motivador por competencia, y que podrían evitar el } \\
\text { abandono del curso. }\end{array}$ \\
\hline
\end{tabular}


Barata, Gama, Jorge y Gonçalves. Domínguez, Saenz-de-Navarrete, De-Marcos, Fernández-Sanz, Pagés, y Martínez-Herráiz, 2013; Ibanez, Di-Serio y Delgado-Kloos

\section{Bunchball y Educause}

\section{Domínguez}

Falkner y Falkner

\section{Hamari y col}

\section{Barata y col}

\section{Seaborn y Fels}

2013 De acuerdo con estos investigadores, cada vez más autores 2014 deciden estudiar la implementación de estrategias de gamificación para involucrar a los estudiantes y promover el propósito de aprendizaje de los estudiantes.

2010 Las insignias, las medallas, los trofeos, los íconos, los

2011 logotipos e imágenes podrían traducir la idea de obtención de un logro, de cumplimiento de una actividad con éxito.

2013 La gamificación podría llegar a obstaculizar la participación de los estudiantes. El diseño y la implementación de los procesos de gamificación deben ser significativos para los usuarios para así lograr garantizar su efectividad.

2014 Sin evidencia suficiente para poder afirmar que el uso de la insignia podría mejorar el compromiso de los estudiantes en un sistema de aprendizaje y que este podría impactar la efectividad de aprendizaje.

2014 Hamari y sus compañeros de investigación argumentan que los estudios actuales se dispersaron, salieron de enfoque y hace falta una evidencia coherente sobre la efectividad de la gamificación en estos entornos. Indagan: ¿los puntos, insignias y rankings podrían llegar a aumentar el compromiso cognitivo de los estudiantes?

2013 Estudio de cinco años con la intención de identificar si al introducir elementos considerados provenientes de la gamificación era posible mejorar la performance en cuanto a la asistencia, participación y aprendizaje de estudiantes en un curso en línea. Declaran no haber encontrado un considerable aumento en la asistencia al curso por parte de los registrados, sin embargo, sí un crecimiento considerable en las publicaciones en el foro. Los investigadores también encontraron que el puntaje final promedio de los estudiantes que realizaron sus estudios en ambientes gamificados han tenido calificaciones mayores que los estudiantes que hicieron el curso cuando todavía no tenía elementos de gamificación.

201532 estudios que se dedicaban a investigar cómo la utilización de elementos de gamificación digital impactaron de manera pedagógica cursos en línea. De los 32 estudios, 20 presentaron resultados positivos que relacionan la gamificación con mayores niveles de motivación y compromiso en las plataformas. 


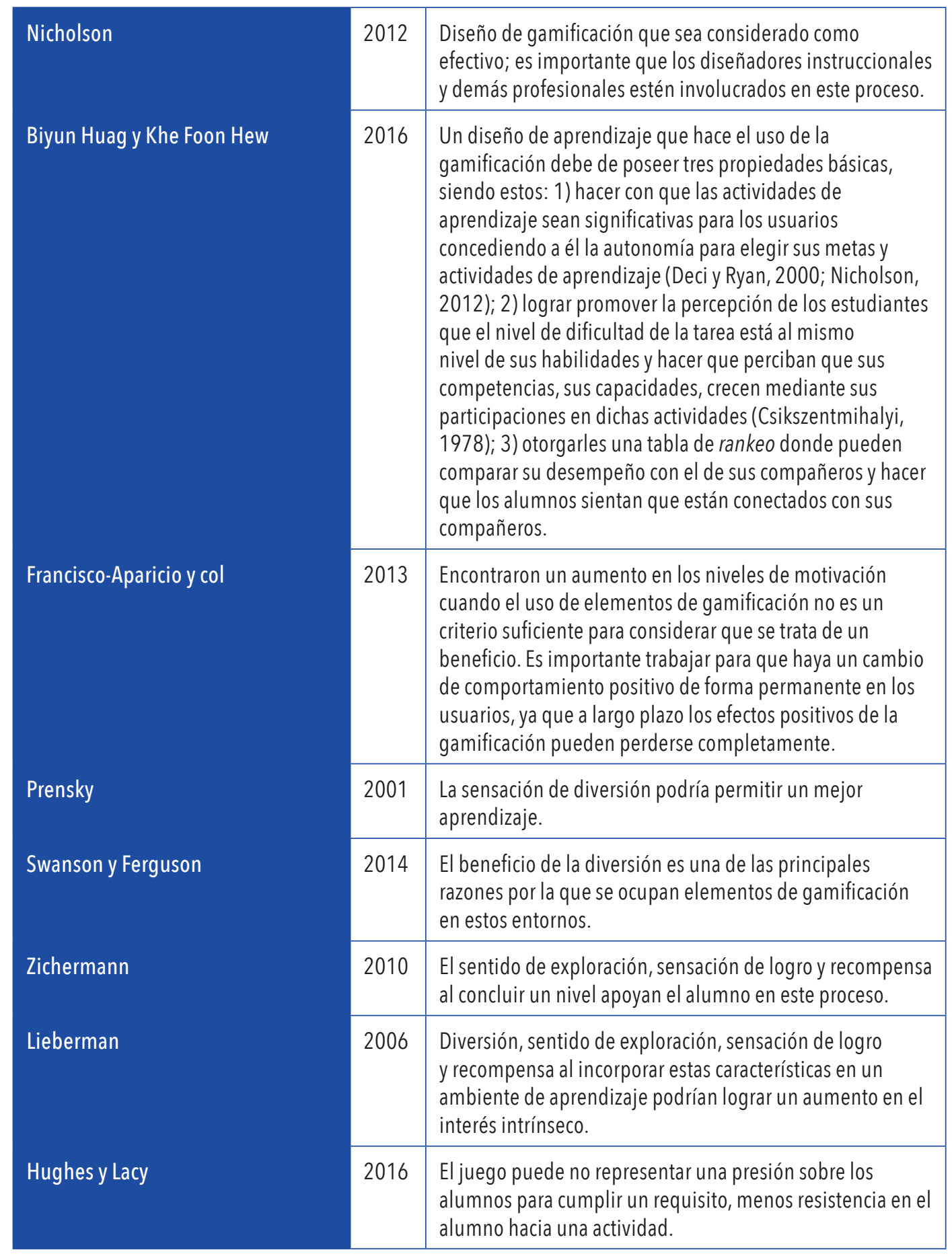




\begin{tabular}{|c|c|c|}
\hline Gee & 2003 & $\begin{array}{l}\text { Los elementos de juego deberían permitir que los } \\
\text { estudiantes trabajen de forma más colaborativa y haya más } \\
\text { trabajo en equipo. }\end{array}$ \\
\hline Zhang y Clear & 2015 & $\begin{array}{l}\text { Cualquier diseño instruccional que ocupa características } \\
\text { de la gamificación necesita proporcionar una mayor } \\
\text { colaboración entre los estudiantes, y destacan que aquellos } \\
\text { que logran éxito ayudan con el surgimiento de conductas } \\
\text { positivas de aprendizaje. }\end{array}$ \\
\hline Kim y col & 2015 & $\begin{array}{l}\text { Cuando estudiantes pasan a ser jugadores, estos colaboran } \\
\text { y participan en una actividad compartida y orientada al } \\
\text { logro de objetivos. }\end{array}$ \\
\hline Hanus y Fox & 2015 & $\begin{array}{l}\text { En un entorno gamificado fallar no es retroceder, se ve } \\
\text { como una oportunidad para aprender de los errores y así } \\
\text { corregirlos. }\end{array}$ \\
\hline Herzig et al. & 2015 & $\begin{array}{l}\text { Los estudiantes que recibieron comentarios constructivos } \\
\text { después de haber fallado en una actividad, pudieron } \\
\text { experimentar una reacción positiva en lugar de sentirse } \\
\text { fracasados. }\end{array}$ \\
\hline
\end{tabular}

FUENTE: ELABORACIÓN PROPIA.

\section{CONCLUSIONES}

Los elementos de gamificación deben de estar relacionados con las características de los usuarios. Las características individuales, como la necesidad de logro, enfoque a objetivos, desarrollo personal, autoeficacia, sus creencias, dificultades informáticas einclinación hacia mecanismo del juego pueden proporcionar un papel relevante en este proceso de experiencia en una plataforma de aprendizaje en línea (Agarwal y Prasad, I999; Kuhlman y Marshello, I975; Martocchio y Webster, I992; Thiebes et al., 2014; Zweig y Webster, 2004).

Es interesante en este contraste de estudios identificar que la gamificación incluso puede tener efectos contrarios, construyendo un sentido de ansiedad y frustración en el usuario si está mal planeada, conduciendo al estudiante a un fracaso, dañando su autoestima y llevándolo a una falta de interés e incluso desear volver a participar de un juego (Malone, 1980). Para Francisco-Aparicio y col (2013), encontrar un aumento en los niveles de motivación cuando hay el uso de elementos de gamificación no es un criterio suficiente para considerar que se trata de un beneficio. Es importante trabajar para que haya un cambio de comportamiento positivo de forma permanente en los usuarios, ya que a largo plazo los efectos posi- 
tivos de la gamificación pueden perderse completamente. En este sentido, sería adecuado realizar un estudio longitudinal con la intención de obtener una captura de los efectos a largo plazo con la relación entre la motivación y la gamificación.

En líneas generales, los estudios en cuanto a la introducción de elementos de gamificación apuntan que se necesitan más análisis longitudinales para comprobar la efectividad de la aplicación a largo plazo. Cuando contrastado con experiencias de aprendizaje no gamificadas se encuentran resultados positivos.

Motivación y participación quizá sean las dos palabras claves más encontradas en cualquier estudio que plantea el uso de gamificación en ambientes de aprendizaje.

\section{REFERENCIAS}

Barata, G., Gama, S., Jorge, J., y Gonçalves, D. (2013). Improving Participation and Learning with Gamification. INESC-ID/IST/Technical, University of Lisbon, Lisbon, Portugal.

Bonwell, C. C. y Eison, J. A. (199I). Active Learning: Creating Excitement in the Classroom. ASHEERIC Higher Education Report, Washington DC: School of Education and Human Development, George Washington University.

Dichev, C. y Dicheva, D. (2017). Gamifying education: what is known, what is believed and what remains uncertain: a critical review. International Journal of Educational Technology in Higher Education.

Dicheva, D., Dichev, C., Agre, G., y Angelova, G. (2015). Gamification in Education: A Systemic Mapping Study. Educational Technology \& Society (I8), 75-88.

Domínguez, A., De Marcos, L. y Fernández, L. (2013). Gamifying Learning Experiences: Practical Implications and Outcomes. España: Computer Science Department, University of Alcalá.

Francisco, A., González, J., Gutiérrez, F., Isla, J. (2012). Analysis and application of gamification. España: Conference: Proceedings of the 13 th International Conference on Interacción Persona-Ordenador.

Prensky, M. (200I). Nativos e Inmigrantes Digitales. Institución Educativa SEK.

Glee, J.P. (2013). What Video Games Have to Teach Us About Learning and Literacy.

García, S., Idrissi, M., Ortega, A. y Gómez, E. (20I8). RIED. Uso de la colaboración y la gamificación en MOOC: un análisis exploratorio. Revista Iberoamericana de Educación a Distancia. http://dx.doi.org/10.5944/ried.21.2.20410

Huang, B. y Hew, K. (2016). Measuring Learners' Motivation Level in Massive Open Online Courses.

Hanus, F. (2015). Assessing the effects of gamification in the classroom: A longitudinal study on intrinsic motivation, social comparison, satisfaction, effort, and academic performance. International Fournal of Information and Education Technology.

Hamari,J., Koivisto,J.,y Sarsa,H. (20I4).Does Gamification Work? - A Literature Review of Emperical Studies on Gamification. 47 th Hawaii International Conference on System Science, 3025-3034. 
Kapp, K. (2012). The Gamification of Learning and Instruction: game-based methods and strategies for training and education. San Francisco: Pfeiffer.

Ibanez, D. y Delgado, K. (20I4). Gamification for Engaging Computer Science Students in Learning Activities: A Case Study. Estados Unidos: IEEE Transactions on Learning Technologies, 7(3), 29I-30I.

Ortega, A., Er, E., Martínez, A., Bote, M.L., Asensio, J.I. y Muñoz, J.A. (2019). Understanding student bebavior and perceptions toward earning badges in a gamified MOOC. Universal Access in the Information Society.

Nicholson, S. (2012). A User-Centered Theoretical Framework for Meaningful Gamification. Paper Presented at Games+Learning+Society 8.o, Madison, WI.

Raed, A. (2018). Do points, badges and leaderboard increase learning and activity: A quasi-experiment on the effects of gamification.

Raed, A. (2017). The Effect of Gamification on Motivation and Engagement. ResearchGate. Recuperado de https://staff.acu.edu.au/-/media/staff-site-rte-docs-only/leo-guides/documents/ icce_20I5_paper_gamification.pdf?la =en \&hash $=96 \mathrm{D}_{3} \mathrm{E}_{5} \mathrm{E}_{9} \circ \mathrm{A}_{17} \mathrm{~B}_{9} \mathrm{~F} 6 \mathrm{~B}_{3} \mathrm{~F} 7 \mathrm{I} 798 \mathrm{E}-$ $5 \mathrm{I}_{4} 8 \mathrm{DA}_{2}$

Liu, D., Santhanam, R., y Webster,J. (2017).Toward Meaningful engagement: a framework for design and research of gamified information systems. Estados Unidos: University of Minnesota. Information and Decision Sciences.

Torres, A., Romero, L., Pérez, M. Staffan, B. (2018). Modelo Teórico Integrado de Gamificación en Ambientes E-Learning (E-MIGA). Revista Complutense de Educación.

Tlili, A. y Chang. M. (2019). Data Analytics Approaches in Educational Games and Gamification Systems. Smart Computing and Intelligence. Singapur: Springer.

Alsawaier, R. (20I8). The effect of gamification on motivation and engagement. The International Fournal of Information and Learning Technology, 35(I), 56-79.

Seaborn, K. y Fels, D. (2015). Gamification in theory and action: A survey. International fournal of Human-Computer Studies, (74), I4-3I. http://dx.doi.org/I0.I0I6/j.ijhcs.20I4.09.006

Shah, D. (2018). By The Numbers: MOOCs in 20I8. Disclosure: Class Central is learner-supported. When you buy through links on our site, we may earn an affiliate commission. Recuperado de https:// www.classcentral.com/report/mooc-stats-2018/ 